\title{
Practice Guidelines Addressing Needs of Pakistani Dentists During the COVID-19 Pandemic Part 2: Recommendations
}

\author{
Samir Riaz Qazi ${ }^{1}$ \\ BDS, FFDRCSI, M.Phil \\ Nurain Rehman ${ }^{2}$ \\ BDS \\ Sameer Nasir ${ }^{3}$ \\ BDS
}

OBJECTIVE: The aim of this article was to develop interim workflow guidelines based on specific needs of dental settings in Pakistan, to help navigate through the COVID-19 pandemic.

METHODOLOGY: Based on needs identified by a survey of 60 dental practitioners, guidelines and protocols were developed after reviewing documents by international and local professional organizations from the fields of dental health care, public health, and hospital epidemiology and infection control.

RESULTS: Recommendations providing details on clinic reorganization, improvement in clinic ventilation, walk-in patient management, PPE use, and environmental infection control, were developed. Protocols have been outlined for performing aerosol generating procedures and for disinfection after procedures.

CONCLUSION: This report supplements the Government of Pakistan guidelines for practicing dentistry during the COVID-19 pandemic. Training of dental health care workers in basic, and in COVID-19 infection control is necessary for adequate implementation of these guidelines.

KEYWORDS: COVID-19, dentistry, infection control, guidelines, ventilation, Pakistan

HOW TO CITE: Qazi SR, Rehman N, Nasir S. Practice guidelines addressing needs of Pakistani dentists during the COVID-19 pandemic part 2: Recommendations. J Pak Dent Assoc 2020;29(S):S14-29.

DOI: https://doi.org/10.25301/JPDA.29S.S14

Received: 23 May 2020, Accepted: 02 June 2020

\section{INTRODUCTION}

$\mathrm{T}$ his report establishes infection control recommendations for essential dental care among different categories of dental practices in Pakistan, during the COVID-19 pandemic. These recommendations supplement the government of Pakistan guidelines ${ }^{1}$ for practicing dentistry during the pandemic, by providing details on environmental infection control, patient management, and safety of patients and dental health personnel; and specifically aim to address the concerns of Pakistani dentists that have not been discussed in other documents published for practice of dentistry during the COVID-19 pandemic. ${ }^{1-8}$ Based on local determinants and cultural dimensions, methods have been introduced to implement infection control measures, which are pragmatic and easy to adopt by Pakistani dentists.

Needs assessment was performed by a field survey of 25 dental practices in Lahore, and an online survey of 35 dental practitioners in Pakistan, published as part 1 of this

1. Consultant Oral Surgeon, Dr. Qazi \& Associates.

2. Advanced Clinical Training Preceptor, UCLA School of Dentistry.

3. Chief Consultant, DentiMed.

Corresponding author: "Dr. Samir Riaz Qazi” < samirqazi@gmail.com > study. The main needs identified were: infection control training of dental health care workers (DHCWs), financial aid and loans, patient awareness and compliance, management of walk-in patients, correct use of PPE and disinfectants, use of high volume suction and rubber dam, economical means of aerosol control and improvement in clinic ventilation and sterilization facilities, and access to genuine and reasonably priced infection control supplies.

The objective of this study was to develop interim workflow guidelines based on specific needs of dental settings in Pakistan to help navigate through the COVID-19 pandemic.

\section{METHODOLOGY}

Results of the needs assessment (part 1 of this study) were used to define and describe three clinic categories, based on infrastructure, facilities, and availability of resources. The Government of Pakistan guidelines ${ }^{1}$ for dental practice during the COVID-19 pandemic were reviewed and areas identified that required further details for adequate implementation, and for addressing the needs identified.

Recommendations and protocols were developed for each of the previously defined clinic categories, after reviewing documents by international and local professional 
organizations from the fields of dental health care, public health, and hospital epidemiology and infection control ${ }^{1-12}$, and by reviewing well designed scientific studies and previous work by the authors. ${ }^{13}$

In the absence of scientific evidence for preventive measures against SARS-CoV-2 spread, certain recommendations are based on strong theoretical rationale, suggestive evidence derived from scientific studies on the spread and control of respiratory viruses having properties similar to SARS-CoV-2, or opinions of respected authorities based on clinical experience.

\section{RECOMMENDATIONS}

These recommendations are not comprehensive, and supplement the Government of Pakistan guidelines for practice of dentistry during the COVID-19 pandemic.

The following categories of dental clinics in Pakistan were defined, and have been used for the recommendations below.

Category A: Clinics confined to one room or hall. The dental surgery/surgeries are not adequately partitioned from the waiting and reception area. Total staff number may be as low as 2, with one dentist and an assistant. Lavatory facilities may not be present or may be shared with other building inhabitants. Minimal infection control facilities are available.

Category B: Clinics with more than one room, where the surgery/surgeries and reception/waiting area have ceiling high partitions with interconnecting doors which may be closed. Lavatory facilities may be shared among patients and staff. Two assistants may be available for each surgery. Basic PPE and infection control supplies are available.

Category C: Clinics with good infrastructure and purpose built facility, able to meet international guidelines.

\section{Clinic reorganization \& infrastructure changes}

1.1 Clinic Zones

1.2 Ventilation of the Dental Clinic \& Surgery

1.3 Patient Management and Flow

1.3.1 Walk-in Patient Management

1.3.2 Scheduled Patient Management

1.3.3 Post Procedure Management

\section{Personal Protective Equipment}

2.1 PPE Guidelines

2.1.1 Entry to Clinic

2.1.2 Exit from Clinic

2.2 PPE for Patient Evaluation \& Non-aerosol Procedures

2.3 PPE for Aerosol Generating Procedures

2.3.1 Minimum PPE requirements for Aerosol Generating
Procedures

2.4 PPE Donning and Doffing

2.5 Seal Check for Respirators

\section{Procedure Infection Control Protocol}

3.1 Protocol for Aerosol Generating Procedures

3.1.1 Guidelines for Aerosol Procedures when Clinic Ventilation is Inadequate

3.2 Protocol for Non Aerosol Generating Procedures

\section{Disinfection}

4.1 Technique for Using Disinfectants

4.2 Selection of Disinfectant

4.3 Protocol for Disinfection after an Aerosol Procedure

4.4 Disinfection of Clinic Environment

4.5 Disinfection of Reusable PPE

4.6 Waste Disposal

4.7 Disinfection of Miscellaneous Items

\section{1- Clinic Reorganization \& Infrastructure Changes}

\subsection{Clinic Zones}

Most clinics require improvement in ventilation, and need to reassign areas in the clinic to facilitate patient flow and procedures during the pandemic (Fig. 1). Infrastructure changes may be necessary to improve ventilation (section 1.2 Ventilation of the Dental Clinic and Surgery), and to add facilities for handwashing, laundry, and showering. For reassignment of clinic areas (Fig. 1), zones may be marked on the floor using a material compatible with frequent disinfection.

Triaging Zone

Seating Area

Donning \& Doffing Zones

Surgery

Storage Area

Dining Area

Changing Area

\section{Triaging Zone}

This area is for screening of both walk-in and scheduled patients.

- For Category A clinics, this area may be outside the door of the clinic, in shade, ideally with a pedestal fan in the summers (Fig. 1).

- For Category B \& $\mathbf{C}$ clinics, this area may be inside, close to the entrance of the clinic.

- Patient information leaflets and posters should be posted in this zone displaying in-office conduct guidelines. Leaflets and SOPs should be laminated for easy disinfection. The following should be included in the leaflets:

- Patients must not be escorted by any attendants, 


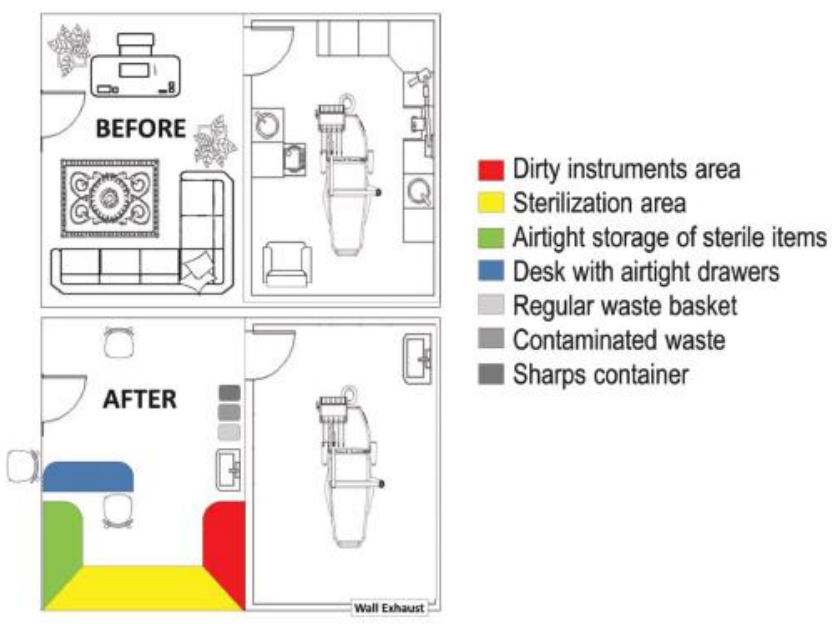

Fig. 1: Reorganization of a single unit Category B clinic. The surgery needs to be cleared of all movable cabinets, stools, furniture, materials, and unnecessary equipment. The reception area needs similar removal of unnecessary items, and may be modified into an instrument processing area, a donning zone created outside the surgery door, and a triaging zone established inside or outside the clinic door. For seating, chairs without armrests should be preferred, setting them apart for maintaining social distance.

with the exception of children, and those needing assistance.

- Patients should leave all extra bags/ belongings in their transport vehicle, if possible.

- Patients should be wearing face masks before entrance into the dental office.

- All patients (walk-in \& scheduled) will be screened for COVID-19 symptoms.

- COVID-19 screening questionnaire, a thermometer and disinfection wipes should be present at the triaging station. A non-contact infrared thermometer is recommended.

- DHCW in this zone should wear a surgical mask and face shield/eye protection. ${ }^{8}$ This PPE is preferable to erecting plastic or plexiglass screens.

- Provide a hand sanitation station at the entrance, with a notice to use it before entry into the rest of the office.

- See section 1.3.1 Walk-in Patient Management, for further information.

\section{Seating Area}

- Category A clinics should instruct the patients to wait in their transport vehicles or in a waiting zone established outside the clinic (Fig. 1). These practices should not have any seating area inside the clinic.

- Category B \& C clinics may follow the same policy but if necessary can place chairs at least 6 foot apart in this area.

- Carpets should be removed from this area; sofas should be replaced with chairs without armrests. Toys, reading material or other communal objects in the clinic should be removed.

- Hand sanitizer should be available.

\section{Surgery}

- The surgery needs to be cleared of all cabinets, stools, furniture and other non-essential removable items.

- All materials, instrument packs, and movable equipment, paper records, electronics, and other items in the dental surgery should be stored/ placed outside the surgery.

- The surgery door should have a self-closing device attached to it.

- For category A clinics all items should be stored in airtight containers, drawers, cabinets or packs.

- All items and surfaces of the entire category A practices, including electronics, will need to be disinfected, and there should be nothing out in the open.

- Paperwork must be limited as much as possible.

- If using paper charting, cover it with a clear barrier so you may read what is needed for appointment and use disposable pens.

- Place chart notes away from the patient contact area when possible.

- Computers \& laptops should be removed from the surgery. If the use of these is absolutely necessary during procedures, barriers may be used. However, surfaces underneath the barriers commonly get contaminated while removing barriers and aerosols may penetrate barriers that are not airtight. Both situations require disinfection of barrier covered surfaces afterwards. Hence, it is best to remove all unnecessary items from the surgery.

- $\quad$ All air-entry or leakage points, other than the desired air entry points, need to be sealed. See section 1.2 Ventilation of the Clinic and Dental Surgery.

- One or two small openings may need to be created into the dental surgery, for air inflow, and to pass materials into the surgery during aerosol procedures.

- This may be a gap between the door and the floor (for air), or via a slit in the lower part of the door (for air and passing items), or through a wall, or through a window in the surgery.

- The size of the opening for air should be governed by the airflow (air changes an hour). See section 1.2 Ventilation of the Clinic and Dental Surgery.

- The opening for passage of items, if 
separate from the air entry point, should be closable with a tight seal.

- Category A clinics should install multiple through the wall exhaust fans in surgery. See section 1.2 Ventilation of the Clinic and Dental Surgery.

- Category B \& C clinics should consider in-line exhausts with ducts collecting, and safely exhausting air outside, which are preferred over through-thewall exhausts. See section 1.2 Ventilation of the Clinic and Dental Surgery.

\section{Donning Zone \& Doffing Zone}

Donning zone: clean area for putting on PPE.

Doffing zone: dirty area for removing PPE.

Both zones require hand hygiene facilities. The doffing zone requires large yellow contaminated waste receptacles.

- For Category A clinics

- The donning zone may be established on the clean side of the clinic where fresh/ new PPE may be stored in sealed cabinets.

- The doffing zone may be established close to the dental unit.

- For Category B \& C clinics

- The donning zone

- may be established in a clean area, outside the dental surgery, either in a dedicated room, or in a corridor on the "clean" side of the surgery door.

- Donning zone may include the clean storage area.

- The doffing zone

- may be established

a. just inside the door of the surgery, or

b. in the corridor outside, on the "dirty" side of the door,

c. or in a separate room.

- The doffing zone should have adequate ventilation and may be located next to the waste disposal area, laundry collection and disinfection area, and showering facility.

- $\quad$ Both the zones may be in the same ante-room to the surgery, with a clean and a dirty side.

\section{Storage Area}

- For category A clinics all sterilised instrument packs, new PPE, and dental materials, and clean items should be stored in airtight cabinets/containers. Instrument packs may be left inside the autoclave to prevent contamination from aerosols.

- Category $\mathbf{B} \& \mathbf{C}$ clinics are likely to already have a separate area for sterilization and storage of clean and sterilized instruments.

\section{Dining Area}

- Consumption of food and drinks should take place in a well-ventilated room of the clinic, not in any of the above areas.

- Social distancing (6 foot distance) must be observed, as masks cannot be worn.

- Break times may be staggered to ensure that social distancing can be maintained in this area.

- Disposable cutlery and crockery may be used, and the waste collected in yellow contaminated waste receptacles.

- Each DHCW should disinfect the surfaces used and touched, before leaving the area.

- The use, and disinfection, of any communal items in this area e.g. tea or coffee making facility, should follow a set protocol based on sound judgement and recommendations for infection control. See section 4 Disinfection.

- Food may be consumed in individual vehicles, or outside the clinic.

\section{Changing Area}

- A changing area may be established where clinic staff can remove street clothing to change into clinic attire i.e. scrubs on entry into the clinic, and vice versa on exit from the clinic. Showering facility may be part of the changing area.

\subsection{Ventilation of the Clinic and Dental Surgery}

Adequate Ventilation: when the dental surgery is in a separate room, which is partitioned from the rest of the dental practice, and there is adequate airflow out of the surgery, so that with the door closed, no aerosol can escape from the dental surgery into the rest of the clinic. Category B \& $\mathbf{C}$ clinics, after implementation of recommendations.

Inadequate Ventilation: when there is no complete partition between the dental surgery and the rest of the practice. Aerosol may diffuse to all parts of the dental practice. Category A clinics. Note: Aerosol procedures should be avoided.

After an aerosol procedure, the door of the dental surgery should remain closed until the aerosol has been eliminated, so that no aerosol escapes to the rest of the clinic. SARS CoV-2 aerosols persist for at least 3 hours if the room is not ventilated. ${ }^{14}$ During these 3 hours the virus will settle on 
surfaces and survive for 2-3 days. ${ }^{14}$

- Adequate ventilation of the dental practice can minimize the risk of infection, and reduce aerosol clearance times. ${ }^{12}$

- Once the aerosol has been eliminated from the room, patients and DHCWs may exit, and cleaning and disinfection may be performed using routine PPE. ${ }^{12}$

For establishing adequate ventilation, air entry and exit points into the dental practice need to be identified, and air flow established in the practice and the dental surgery according to existing infrastructure, needs, preferences, and resources (Fig. 2).

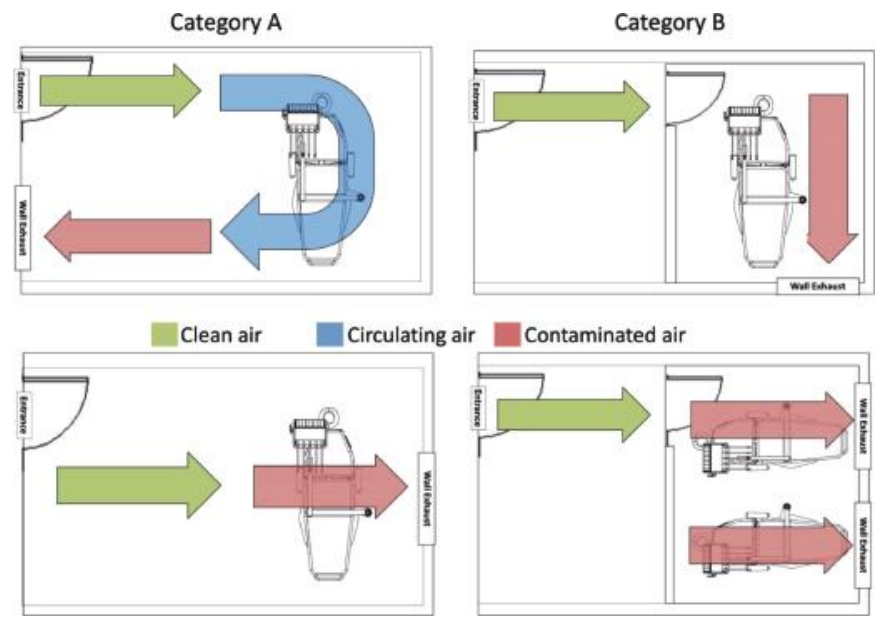

Fig. 2: Generating airflow for improved ventilation in category A \& B clinics, using through-the-wall exhausts. Left top floor plan has one outside wall. Pedestal and wall bracket fans may additionally be employed to direct air flow away from reception and towards the surgery or the outside, and from the dental unit towards the outside. Windows, when present, may be opened for air inflow.

\section{Ventilation for Category A Clinics:}

- For general ventilation of the clinic, these practices should at minimum install exhaust fans (Fig. 2). Windows may be opened if present, and pedestal and wall bracket fans may be used to direct airflow towards the outside.

- Aerosol procedures should be avoided. See section 3.1.1 Guidelines for Aerosol Procedures when Ventilation is Inadequate.

- Practices should consider installing partitions sealing the surgery from the rest of the clinic. Plastic sheets may be used, though it may be best for practices to invest in solid partitions, with doors that have closable openings for air-entry, and item passage.

\section{Ventilation for Category B \& C Clinics}

i. Ventilation of the Entire Clinic

- Use principles for dental surgery ventilation (below) to ensure adequate ventilation and air flow through the entire practice, especially the doffing zone and dining area.

ii. Options for Ventilation of the Dental Surgery a. Through-the-wall exhausts

- The exhaust should be installed so that it is located opposite to the point of air-entry, which is usually through a gap between the door and the floor, or may be established elsewhere e.g. window. ${ }^{9}$

- The door should be kept closed during aerosol procedures, and no air should leak into the rest of the dental practice.

- Install a self-closing device on the door. ${ }^{9}$

- Depending on the air flow rate of the exhaust fan, more than one exhaust fan may be installed in the dental surgery, to ensure more air changes per hour.

- Test by closing the surgery door and:

- Release smoke near the air inlet. The smoke should all get sucked into the surgery.

- Or place a thin strip of tissue paper near the air inlet. The strip should get sucked into the surgery. ${ }^{15}$

- On the outside of the building, the exhaust fan should ideally be located 25 foot away from human traffic and from other air inlets to the building. ${ }^{16}$

\section{b. In-Line Exhausts with Ducts}

- In-line exhausts with ducts are preferred to through-the-wall exhausts.

- The inlet of the duct may be installed 6 inches from the floor, near the foot end or the head end of the chair. Air entry should be from the opposite side of the room. ${ }^{16}$

- On the outside of the building, the exhaust duct outlet should be 25 feet from human traffic and air inlets ${ }^{16}$, and ideally on the roof pointing up. If requirements cannot be met, or if there are health care and environmental concerns, exhaust air may be filtered.

- Seal all other air entry points in the surgery to try and create better airflow and possible negative pressure. E.g. seal electrical sockets, air conditioner grills, leaks from around the windows and other doors.

- Use guidelines above for the door, and for testing. 


\section{c. Other Methods}

Methods that follow may be considered by Category $\mathrm{C}$ clinics, but are NOT recommended for immediate implementation during the pandemic, due to lack of evidence for their use in the control of SARS-CoV-2 transmission. Some of these methods are expensive and impractical to implement, and may have value mostly as marketing tools.

I. HVAC systems with HEPA filters, and Negative Pressure Dental Surgery.

- Medical and dental engineers should be consulted. Requires major infrastructure changes.

- HVAC systems with HEPA filters may be used for disinfection and cooling of all air re circulating in the dental practice, and for all air-entry to the dental surgery. ${ }^{9}$ Note: HEPA filters are not designed for filtration of wet aerosols ${ }^{9}$, and their efficacy for SARS-CoV-2 aerosol filtration is not known.

- Follow guidelines above for exhausting air.

- The dental surgery may be converted to a negative pressure room, with $>12$ air changes an hour. ${ }^{9}$

- Ante-room (at half negative pressure) may be established:

a) For donning and doffing of PPE - with assigned clean and dirty areas.

b) For passage of instruments and equipment during the procedure.

- Aerosol clearance time at 12 air changes per hour is 35 minutes, and at 50 air changes per hour is 8 minutes. $^{12}$

- Use of portable or plug-in air purifiers with HEPA filters may help improve clinic air quality, and may reduce aerosol clearance time after procedures. They may be used when clinic ventilation is not adequate. Note: these devices do not replace the need for adequate ventilation and airflow, and the use of PPE.

II. Aerosol reduction devices

- The use of rubber dam and one or two large bore (at least $8 \mathrm{~mm}$ dia.) high volume suctions should be the primary measures for aerosol reduction. There is no evidence yet to support the use of any other device for COVID-19 aerosol control.

- Aerosol collectors do not reduce the microbial contamination reaching the dentist. Despite their use, contamination is found on the head and attire of the operator and assistant, and the aerosol persists in the room for the usual time, based on ventilation and airflow. ${ }^{17}$

- UV Light: The use of a UVC light may help improve air quality and partially disinfect surfaces after a procedure, or at the end of the day. Use during the aerosol clearance time is not possible due to the presence of personnel in the room. Use afterwards will increase between-patient time, as disinfection will still need to be performed.$^{18}$

- Fogging, commonly with hypochlorous acid, is used in some practices, after all other disinfection procedures, as an additional measure for improving air quality. There is no evidence to support the use of fogging (a room or a person) for control of COVID-19 transmission.

\subsection{Patient Management \& Flow}

\subsubsection{Walk-in Patient Management}

- Screen all patients in the triage zone. For organizing the Triage Zone, see section 1.1 Clinic Zones.

- Ask questions about COVID-19 symptoms and contact with COVID-19 patients (questionnaire), and about the presenting complaint.

- Record temperature.

- Record contact information (for calling patient, follow up, and contact tracing).

- Verbally explain clinic policy for treatment during the pandemic

- If the case is non-urgent, the patient should not be seen immediately, and instead contacted by the dentist for a remote consultation using appropriate communication technology, see section 1.3.2 Scheduled Patient Management.

- If a patient is positive for symptoms of COVID-19, Government of Pakistan dental practice guidelines must be followed (do not treat). ${ }^{1}$

- Ask patients to call and report any illness or systemic symptoms they develop after their visit to the clinic.

- According to clinic policy, hand over:

- Patient information form, consent form, COVID-19 questionnaire, a disinfected or disposable pen, information leaflets on COVID-19 clinic protocols, and a surgical mask if the patient is not wearing one. See Triage Zone, section 1.1 Clinic Zones.

- Ask the patient to wait in their transport or in the waiting area, outside or inside the clinic. See Waiting Area, section 1.1 Clinic Zones.

- While waiting, the patient should be contacted by the dentist over phone for screening and pretreatment counselling. See section 1.3.2 Scheduled 
Patient Management.

- Call the patient when ready.

- Open the door for the patient

- Receive forms, pen, leaflets (and payment, if applicable) in a tray/basket (to be considered as dirty/infected).

- Offer hand disinfectant.

\subsubsection{Scheduled Patient Management}

- All patients who call the clinic for a consultation must initially be scheduled for a remote dentistry consultation.

- This remote dentistry session includes:

- Screening patients for COVID-19 by asking relevant questions.

- Patient evaluation using audio-visual means, and categorizing urgency of treatment.

- For non-emergency cases, advice may be given on the phone.

- If the patient requires urgent treatment:

- Schedule appointment.

- Brief the patient about information relevant to their treatment including cost, pre \& post-procedure instructions and follow up (inform if they develop COVID-19 symptoms), clinic entry and exit protocol, and take verbal informed consent.

- Document all communication in the patient record.

- Appointments for patients must be spaced out such as to avoid patients waiting in the seating area, keeping in mind the time required for disinfection of the surgery.

- If clinic ventilation is not adequate, schedule one aerosol generating procedure at the end of the day. See section 3.1.1 Guidelines for Aerosol Procedures when Clinic Ventilation is Inadequate.

- Patients should be encouraged to make payment electronically when possible.

\subsubsection{Post Procedure Patient Management}

- All post-op instructions should be given to the patients prior to the start of the procedure, i.e. during their remote consultation or after triaging, to minimise contact with the dentist and dental staff post-procedure.

- After an aerosol generating procedure (AGP)

- Patients may exit the dental surgery after the minimum aerosol clearance time, when ventilation is adequate. ${ }^{12}$ In category $\mathbf{A}$ clinics, exit should be as soon as possible. See section
3.1.1 Aerosol Procedures when Ventilation is not Adequate; for aerosol clearance times, see 1.2 Ventilation of the Clinic and Dental Surgery.

- Patients should remove any PPE they are wearing during the procedure before exiting the surgery, or in the doffing zone. See Doffing Zone, section 1.1 Clinic Zones.

- Patients should perform hand hygiene before exiting the clinic.

- Door should be held open for patients on exit.

\section{2- Personal Protective Equipment}

The most efficacius PPE items against COVID-19 are masks and eyewear, as these items protect the nose, mouth and eyes, which are the routes of entry for the virus. N95 or better respirators, and airtight eye protection, e.g. goggles, are recommended for all patient contact.

\subsection{PPE Protocol}

\subsubsection{Entry to the Clinic}

- Patients should be asked to

- Wear a surgical mask, or follow strict respiratory etiquette i.e. cough or sneeze into elbow or sleeve.

- Optionally, according to availability and clinic protocols, patients may be asked to wear gowns and shoe covers/shoes, head caps.

- For aerosol generating procedures (AGPs), patients should be asked to wear a gown and protective eyewear, which should be removed just before leaving the dental surgery, or in the doffing zone. See Doffing Zone, section 1.1 Clinic Zones.

\section{Staff members should}

- Change into work clothes e.g. scrubs, or wear a gown on top of regular clothing.

- Change shoes to closed work shoes, made of material which may be sprayed with liquid disinfectant when necessary. If not changing shoes, disinfect street shoes including soles.

- Wash hands.

- Wear a surgical mask, which should be worn all day.

- When staff members are in close proximity to patients (e.g. triaging zone), eye protection should be used.

- Gloves do not need to be worn routinely in the clinic. Instead, frequent hand hygiene is critical, along with disinfection of shared items (computers, phones) and commonly touched surfaces. See section 4.4 Disinfection of Clinic Environment. 


\subsubsection{Exit from the Clinic}

Work clothes and shoes should be considered infected, and need to be disinfected before routine washing.

- Staff Members should change into street clothes.

- Work clothes should be

- placed in a designated bucket/receptacle for disinfection,

- or sealed in a plastic bag to take home for disinfection (this is not recommended, and every clinic should provide laundry service for work clothes)

- The outside of this bag should be disinfected, and hands washed.

- Another option is to go straight home and remove the contaminated clothes for disinfection, and take a shower, but the transport used may get contaminated and will need to be disinfected.

- Wearing work clothes when using public transport is not recommended.

\subsection{PPE for Patient Evaluation and Non Aerosol Procedures}

Generation of aerosols is not only limited to dental AGPs but may occur any time the patient coughs or gags. - History Taking:

- The dentist and patient should wear surgical masks and, if possible, protective eyewear.

- The dentist should stand at the 7 or 8 o'clock position during history taking, ideally 6 feet away.

- Dental Examination and Non-Aerosol Procedures:

- Wear N95 respirator. If disinfecting and reusing respirators, a disinfected respirator may be used. ${ }^{8}$ See section 4.5 Disinfection of reusable PPE.

- When N95 respirators are not available, a surgical mask may be used in an emergency, with a face shield and eyewear. ${ }^{19}$

- Protective Eyewear is recommended for both the patient and dentist.

- Gowns and head caps or other outer attire may be used according to clinic policy.

- If outer attire/gown is not being changed between patients, attire should be considered infected, and all effort should be made to prevent the attire contacting the next patient, or other surfaces in the clinic.

- If the mask and eyewear are not being changed between patients, at minimum, wash hands after a procedure, adjust the mask and eyewear, wash hands again, and then wear new gloves for the next patient.

\subsection{PPE for Aerosol Generating Procedures (AGPS)} AGPs include:

- Ultrasonic scaling and polishing

- Air Polishing and abrasion, prophy jets, rubber cups

- Any procedures involving use of air-water (triple) syringe

- Use of slow and high speed rotary instruments

- Tooth preparation (Crowns, Bridges, Fillings, and Veneers etc.)

- Root canal opening

- Sectioning of teeth for extraction using burs

- Dental implant placement

- Denture adjustments, when contaminated with saliva

- Sharp edge reduction

- Air abrasion

- Procedures involving lasers

The principle for risk reduction remains the same: protect the mouth, nose and eyes, which are portals of entry for the virus. Everything else (skin, hair, attire, shoes) may be carefully disinfected after the procedure.

\subsubsection{Minimum PPE Requirements for AGPS:}

i. Mask: N95 or better respirator.

- Over masking with a surgical mask may be done to protect the N95 respirator from droplets.

- Check fit and perform user seal check. See section 2.5 Seal check for Respirators.

ii. Eyewear:

- Protective eyewear with side protectors, and face shield.

- If possible use eyewear with airtight seal (e.g. goggles), in which case face shield is not essential, but is still useful as it will protect the mask and face from droplets.

\section{iii. Other PPE:}

- Wear a gown and head cap, or coveralls, and/or other PPE according to clinic policy.

- Wear shoe covers, or disinfect shoes before exit from surgery.

- Gowns protect the inner attire from large droplets and some aerosol. After removal of outer PPE, inner attire, skin and hair should still be considered contaminated.

\subsection{PPE Donning and Doffing}

\section{PPE Donning (wearing) Sequence ${ }^{20}$ :}

1. Perform hand hygiene.

2. If double gloving, put on gloves. 
3. Put on shoe covers (if applicable).

4. Put on a gown.

5. Put on a mask/respirator.

6. Put on eye protection.

7. Put on a head cap (if applicable).

8. Perform hand hygiene.

9. Put on gloves.

\section{PPE Doffing (removing) Sequence ${ }^{20}$ :}

1. If double gloving, remove outer gloves.

2. Remove shoe covers (if applicable).

- Or disinfect shoes including soles, on exiting the dental surgery.

3. Remove head cap (if applicable).

4. Remove gown and gloves together.

- Break the ties at the neck by pulling on the upper front portion of the gown with the hands still gloved, balling or rolling in the contaminated surfaces, and pulling the gloves off inside-out as the hands are withdrawn from the gown sleeve.

- The gown and gloves can then be placed in a disposal receptacle together.

5. Perform hand hygiene.

6. Remove eye wear.

7. Remove mask/respirator.

8. Perform hand hygiene.

\subsection{Seal Check for Respirators}

a. Positive Pressure Seal Check ${ }^{21}$

Conducted on respirators without exhalation valves.

- Once the respirator is properly donned,

1. Place your hands over the face piece, covering as much surface area as possible.

2. Exhale gently into the face piece.

- A slight positive pressure should build up inside the face piece without any evidence of outward leakage of air at the seal.

- Signs of leakage:

- Feeling of air movement on your face along the seal of the face piece.

- Fogging of your glasses.

- Lack of pressure being built up inside the face piece.

b. Negative pressure Seal Check ${ }^{21}$

Conducted on respirators with exhalation valves. May be used for respirators without exhalation valves.

- $\quad$ Once the respirator is properly donned
1. Cover the filter surface with your hands as much as possible and then inhale.

2. The face piece should collapse on your face and you should not feel air passing between your face and the face piece.

In case of unsatisfactory positive or negative seal check $^{21}$

- Use both hands to readjust the nosepiece by placing your fingertips at the top of the metal nose clip.

- Slide your fingertips down both sides of the metal strip to more efficiently mould the nose area to the shape of your nose.

- Readjust the straps along the sides of your head until a proper seal is achieved.

\section{3- Procedure Infection Control}

Note: protocols always need modification according to local circumstances, resources, and feasibility.

\subsection{Infection Control Protocol for Aerosol Generating Procedures (AGPS)}

- Absolute requirements for AGPs:

i. Adequate ventilation of the dental surgery. See section 1.2 Ventilation of the Dental Clinic and Surgery.

ii. $\quad$ N95 or better respirators and eye protection. See section 2 Personal Protective Equipment.

iii. Rubber dam, if applicable. ${ }^{22}$

iv. High volume suction. ${ }^{23}$

- Before patient entry, aseptically set up the instrument tray, load LA syringe, dispense any materials needed during the procedure (e.g. on a glass slab, dappen dish). Apply barriers, according to clinic policy.

- If there is adequate ventilation (section 1.2 Ventilation of the Dental Clinic and Surgery), any other items that are needed during the procedure may be placed on a trolley outside the operatory door (or in the corridor, or in an ante-room) along with bins for disposal of waste and reusable attire (e.g. gowns).

Dentists and assistants should don PPE in the donning zone i.e. designated clean area of the clinic. See sections 1.1 Clinic Zones \& 2.3 PPE for Aerosol Generating Procedures \& 2.4 PPE Donning and Doffing.

- Patients should be given a gown and eye protection to wear.$^{23}$

- Patients should be asked to perform preprocedural mouth rinse using

a. $1 \%$ hydrogen peroxide for 60 seconds or b. $0.2 \%$ povidone-iodine for 30 seconds. ${ }^{24}$

- High volume suction should be held 6 to $15 \mathrm{~mm}$ 
from an aerosol generating device ${ }^{25}$, using an appropriate suction tip with at least an $8 \mathrm{~mm}$ wide opening. ${ }^{25}$

- After the procedure has started,

- Bottles and tubes of materials should not be opened inside the aerosol room.

- Any further materials and items needed should be handed or dispensed by a second assistant outside the room via the air entry point, or by briefly, partly opening the door.

- When aerosol generation ends, time should be noted.

- Patients may exit the surgery, after waiting for aerosol clearance time (section 1.2 Ventilation of the Dental Clinic and Surgery), removing PPE in the doffing zone. See Doffing Zone, section 1.1 Clinic Zones.

- DHCWs should dispose of contaminated waste and sharps before leaving the room, and remove shoe covers or disinfect shoes at exit.

- All PPE, except mask and eye protection, should be removed before leaving the surgery, or removed in a designated doffing (dirty) area of the clinic. See PPE Doffing Sequence, section 2.4 PPE Donning and Doffing.

- For disinfection of the dental surgery after an aerosol procedure, see section 4.3 Protocol for Disinfection after an Aerosol Procedure.

\subsubsection{Guidelines for Aerosol Procedures when Clinic Ventilation is Inadequate}

If clinic ventilation is inadequate (section 1.2 Ventilation of the Dental Clinic and Surgery), aerosol procedures should not be performed. ${ }^{1}$ In case an aerosol procedure needs to be done in emergency:

- Procedure should be scheduled/performed at the end of the working day.

- Only the patient, dentist and assistant/s (all using appropriate PPE, see section 2.3 PPE for Aerosol Generating Procedures) should be present in the entire dental practice during the procedure. All other persons must vacate the clinic.

- $\quad$ Planning is necessary to dispense all materials and items needed for the procedure before the generation of aerosol.

- All material bottles and other loose items should then be stored in airtight containers, so they do not need disinfection later.

- Any leftover dispensed materials at the end of the procedure must be discarded.

- If any bottle/tube/container is opened during the procedure, the entire material/item should be considered contaminated.

- The patient and all staff should leave the clinic as soon as possible after an aerosol procedure, removing mask and eyewear immediately before exit.

- Clinic exhausts should be left turned on.

- DHCWs attire, shoes, hair and skin at exit should be considered contaminated. Disinfect hands and wear a clean mask if possible. Avoid touching the mouth, nose and eyes.

- The clinic should not be entered for at least 3 hours after the procedure.

- Upon entry (the next day) or after at least 3 hours, the entire dental practice environment must be disinfected thoroughly, because during the first few hours aerosol will settle on all clinic surfaces and the virus may survive on these surfaces for 3 days.

\subsection{Infection Control Guidelines for Non-Aerosol Generating Procedures}

- Non-aerosol procedures, even routine examinations, may generate aerosols if the patient coughs or gags. ${ }^{26}$

- Protocols generally similar to aerosol generating procedures should be used.

- The patient is not required to wear any PPE during non-aerosol procedures.

- For PPE and attire recommendations, see section 2.2 PPE for Patient Evaluation \& Non-aerosol Procedures.

- Disinfection of the dental surgery after the procedure may be limited to the dental unit, and other commonly touched surfaces.

\section{4- Disinfection}

For disinfection to be effective, the surface must be exposed to the disinfectant for the required time. This usually means that the surface needs to stay wet with the liquid disinfectant.

\subsection{Technique for Using Disinfectants}

- Wear gloves. If using sprays, wear a mask and eye protection.

- Clean visible contamination on the surface using a 
disposable wipe, damp with soap solution or disinfectant.

- Apply the disinfectant using a disposable cloth or wipe soaking in disinfectant.

- The surface may be sprayed and a gloved hand (or wipe wet with disinfectant) used to spread the disinfectant.

- Ensure that the entire surface stays wet with the disinfectant for the recommended time.

- For the floor, a mop soaked in disinfectant solution may be used to spread the disinfectant for the required time. Sprays are not as effective as a mop for floor disinfection. ${ }^{27}$

\subsection{Selection of a Disinfectant}

- From the disinfectants available, each practice may select 2 or 3 disinfectants for daily use, according to their needs.

- In addition to the disinfectants discussed underneath $^{28}$, any intermediate level disinfectant, with tuberculocidal claim, may be used for surfaces, instruments, and equipment disinfection, following manufacturer instructions.

a. Soap solution

- Soap or detergent solutions can destroy coronaviruses, but the surface requires rubbing with the solution, which is impractical for routine use.

- Use for:

- Hand washing.

- Cleaning surfaces before disinfection.

- Routine instrument cleaning after disinfection.

- Laundry of attire.

b. Sodium Hypochlorite

- Available commonly as 5.25\% household bleach. $12-15 \%$ concentrations are available.

- Wear mask, gloves, and eye protection while preparing, and during use.

- Is corrosive to metals; do not immerse instruments for more than 10 minutes, and do not use routinely on metal surfaces.

- Use as:

- $0.1 \%$ solution to disinfect instruments and other items by immersion for up to 3 to 5 minutes. ${ }^{29}$ Rinse instruments prior to immersion in sodium hypochlorite solution and immediately after removal from the solution.

- $0.1 \%$ solution for disinfection of surfaces and floors. Surfaces should stay wet for
1 minute. ${ }^{29}$

- $0.05 \%$ solution for disinfection of attire by immersion. Soak thoroughly and leave immersed overnight or at least 30 minutes. ${ }^{30}$

- $0.5 \%$ solution for waste disinfection. ${ }^{30}$

c. Hydrogen Peroxide

- Available commonly in 1-6\% concentration.

- After application on surfaces, it is non-toxic to skin, and is compatible with many impression materials and common surfaces of the dental surgery.

- Use as

- $1 \%$ solution for surface disinfection. Exposure time: $1 \mathrm{~min}$.

- $1 \%$ solution for mouth rinse, for one minute.

- $3 \%$ solution for disinfection of items by immersion, for at least one minute. ${ }^{24}$

d. Alcohol

- Generally used in combination with other disinfectants for surface disinfection. Available as wipes, sprays, gels, hand disinfectants, and as isopropyl alcohol. Non-toxic to skin, and compatible with most surfaces.

- $70 \%$ solution is effective for surface disinfection when applied for 1 minute. ${ }^{31}$

- It is useful for disinfection of hands and gloves, surfaces of the dental unit, and for items like curing lights, bottles, tubes, shoes.

- Alcohol fixes bio burden and dust onto surfaces and is not ideal for routine use as a surface disinfectant.

- In hot environments, alcohol may evaporate while being sprayed, and from the surface being disinfected, before the recommended contact time is over. Especially when concentrations higher than $70 \%$ are used.

- If using alcohol, clean surface thoroughly first, followed by liberal application and spreading.

\subsection{Protocol for Disinfection after an Aerosol Procedure}

- Start disinfection of the dental surgery after aerosol clearance time is over, which is 3 hours when ventilation is not adequate. See section 1.2 Ventilation of the Clinic and Dental Surgery.

- An intermediate level disinfectant nontoxic to human skin, non-corrosive to metals, and compatible with the dental unit upholstery, is needed for all commonly touched surfaces, and for the dental unit. 
- For floors and other uncommonly touched surfaces, sodium hypochlorite or other intermediate level disinfectants, may be used, following manufacturer recommendations.

- Protocol:

- Wear a surgical mask, gloves and protective eyewear.

- Clean all commonly touched areas and all visible contamination using soap solution and disposable wipes. Paying attention to the spittoon and surrounding area.

Paying attention to the spittoon and surrounding area.

- Remove all handpiece tubing and the triple syringe from their holders, hold everything over the spittoon and run water through all the tubes for 30 seconds. ${ }^{11}$

- Before replacing the tubes and syringe, disinfect the holders first, and then wipe one tube at a time with a disposable wipe wet with the disinfectant, followed by spraying all tubes, connectors, and syringe thoroughly with the disinfectant.

- Disinfection of the dental unit tray, arms, light, entire seat, assistant side suction holders and tubing, and the dental unit tubing may follow in a set routine, using disposable wipes wet with the disinfectant and/or spray.

- Surfaces must stay wet with the disinfectant for the recommended time.

- All other surfaces of the dental surgery, within reach, need similar disinfection. For e.g. counters, cabinets, doors, handles, stools, sharp boxes, waste baskets, trolleys, bottles, $\mathrm{x}$-ray units, computers, screens and all other surfaces and items in the room, including walls and windows. It is preferable to remove all unnecessary items from the surgery to make this task easier.

- If surfaces or items not in routine use have been covered by an airtight barrier, the barrier may be cleaned and disinfected without having to change it between patients.

- Disinfect the floor at the end, starting from a corner away from the door and ending by cleaning the area inside and outside the door.

- Soles of DHCW shoes should be wet with the disinfectant, or use a disinfectant spray on the shoes.

- $\quad$ Remove PPE on exit from surgery and wash hands.

\section{Dental Unit water cleaning}

Ensuring quality of dental unit water reduces bioburden in aerosols. Dental unit waterlines promote bacterial growth and development of biofilm due to the presence of long narrow-bore tubing, inconsistent flow rates, and the potential for retraction of oral fluids from hand pieces into the unit tubing. ${ }^{32}$

- Ensure quality by intermittent or continuous treatment (disinfection), always according to the dental unit manufacturer's recommendation. Examples:

a. Continuous Treatment

- Use $0.02 \%$ hydrogen peroxide solution ${ }^{33}$, or $0.12 \%$ chlorhexidine ${ }^{32}$ as dental unit water.

b. Intermittent Treatment

- Weekly 0.5\% Sodium Hypochlorite Shock Treatment ${ }^{34}$ :

1. Detach the unit's water bottle, add bleach solution to it and then re attach the bottle to the unit.

2. Run the bleach solution into each line

3. Run each scaler, handpiece, and airwater syringe line for 10 seconds or until you smell the bleach flow out the spout or you notice the "slippery" feel of the bleach.

4. After 10 minutes, detach the water bottle, dump the remaining solution into the sink, and refill with warm water.

5. Flush the lines for 1-3 minutes or until the remaining bleach odour disappears.

- After procedures, discharge water from the waterlines of handpieces, scalers and air-water syringes by operating the devices for 30 seconds. ${ }^{32}$ See section 4.3 Protocol for Disinfection after Aerosol Generating Procedures.

- For sterile procedures (e.g. oral surgery), sterile irrigants (e.g. saline) should be used. Delivery systems should have sterile water lines/tubes, either disposable or autoclavable. ${ }^{32}$

\subsection{Disinfection of Clinic Environment}

- Identify all commonly touched surfaces in the clinic, and list in a logical sequence for disinfection. Surfaces include door handles, areas touched on door frames, furniture, counters, light switches, water dispenser, sinks, fixtures, soap dispensers, thermometer.

- Prepare a trolley with supplies, or have available: 
- One or two disinfectants in spray bottles. See section 4.2 Selection of Disinfectant.

- Disposable wipes

- Gloves

- Waste basket

- Floor wiper

- Train all DHCWs in the clinic on disinfection protocols, and assign one DHCW to disinfect all listed surfaces multiple times a day (at least twice daily), using a disinfectant non-toxic to human skin.

- Relevant surfaces must be disinfected after entry and exit of every patient and staff member.

- If present, the toilet must be disinfected after every use. DHCWs may be trained to disinfect the toilet themselves after use.

- Wear a mask, gloves and eye protection during disinfection.

- All visible contamination should be washed or cleaned, and all surfaces of the toilet, including floor, liberally sprayed with a disinfectant.

- If using sodium hypochlorite, or other disinfectant toxic to skin, after the required disinfection time, wash and/or wipe-clean the toilet seat, tap fixtures and other commonly touched surfaces using disposable wipes.

- The floor should be rinsed and wiped at the end.

- At the end of the day, all dental practice floors need disinfection, along with all furniture and surfaces identified previously.

- In poorly ventilated clinics, the day should start with complete dental practice disinfection. See section 3.1.1 Guidelines for Aerosol Procedures when Clinic Ventilation is Inadequate.

\subsection{Disinfection of Reusable PPE}

i. Masks and Respirators

All surgical masks and single use respirators (e.g. N95) should ideally be used once and disposed of.

There is no clear recommendation on the reuse of surgical masks. The use of liquid disinfectants reduces filtering efficiency of these masks, as does autoclaving, and these methods should not be used. Disinfection with time (stored in a paper bag for one week) may be attempted in case of non-availability. All surgical masks that get wet during use should be discarded. ${ }^{8}$

In the situation of shortage of N95 masks during the pandemic, and need to provide urgent care, CDC recommends different 7 methods of disinfection of respirators. ${ }^{8}$ Two are discussed below.

Precautions when Using "Disinfected" respirators":

- The outside of the mask should be considered contaminated. Wear gloves when putting on, and/or perform hand hygiene after touching or adjusting the mask.

- Avoid touching the inside of the mask.

- Visually inspect the respirator to determine if its integrity has been compromised.

- Check that respirator components such as the straps, nose bridge, and nose foam material did not degrade, which can affect the quality of the fit, and seal.

- Perform a user seal check after putting on. See section 2.5 Seal Check for Respirators.

- If the integrity of any part of the respirator is compromised, or if a successful user seal check cannot be performed, discard the mask and try another.

For aerosol procedures, it is recommended that a new N95, or better respirator, be used. Disinfected N95 respirators should be reserved for non-aerosol procedures. ${ }^{8}$

\section{Methods of N95 and Respirator Disinfection}

Do not use autoclave, dry heat, alcohol, soap, bleach and dry microwave radiation. ${ }^{19}$

- For reusable respirators (e.g. P100), manufacturer instructions should be followed for disinfection.

- Disinfection Methods for Single Use Respirators include:

a. Time

- Corona virus lasts on masks for 5-7 days. ${ }^{35}$

- Procedure:

1. Label a paper bag with user name and date.

2. After use, place the respirator in the paper bag, held open by an assistant.

3 . Wash hands.

4. Store in a well-ventilated area for at least one week.

5. Reuse after one week.

- Should be re-used only by the labelled user, following precautions above.

- Do not re-use more than 5 times.

b. Hydrogen Peroxide (H202)

Some respirators may be suitable for disinfection by immersion in liquid (3-6\%) hydrogen peroxide for at least one minute. $\mathrm{CDC}$ has listed the respirators that may be 


\section{ii. Washable Gowns and Attire}

disinfected safely with hydrogen peroxide. ${ }^{8}$

Washable gowns and attire may be reused after disinfection and laundry. Disinfect by immersion in $0.05 \%$ sodium hypochlorite solution for at least 30 minutes, followed by rinsing and routine laundry. ${ }^{30}$

iii. Reuse of Eyewear and Face Shields

- If not changing eye protection between patients, consider the eyewear and face shield infected, and whenever touched, perform hand hygiene, or change gloves.

- After use, eyewear should be washed with soap and water. If not using a disinfectant, wash thoroughly with soap and water, rubbing or brushing all surfaces for 1-2 minutes to create foam/bubbles.

- If using a disinfectant; wash, rinse, dry with a disposable tissue, and apply the disinfectant using spray, wipes, or immersion. The disinfectant may be rinsed off after the required time, especially if using sodium hypochlorite or another disinfectant toxic to skin. After cleaning and disinfection, antimist sprays or other coatings may be used.

\section{iv. Loupes}

Standard loupes may be difficult to wear with current PPE recommendation, and if used, manufacturer guidelines should be followed for disinfection between patients.

\section{v. Head cap/protection}

Reusable plastic head-caps may be washed and disinfected like the eyewear. However, single use disposable head coverings are preferred.

\subsection{Waste Disposal}

- Waste collection in the dental clinic should be in:

- Plastic bag lined containers:

- Waste receptacle for non-contaminated waste.

- Yellow baskets for contaminated waste.

- Sharp containers: sealable puncture-resistant containers (thick plastic) with a biohazard label, located at the point of use, for collection of needles, scalpel blades, syringes etc.

- Collected contaminated waste should be sealed in leak-resistant biohazard bags and sent for incineration; along with all 2/3rd full sharp containers. ${ }^{11}$

- All clinic waste being disposed of as garbage should be disinfected first.

1. Wear gloves, mask and protective eyewear.

2. Do not overfill the garbage disposal bag.
3. Liberally spray the garbage, inside the disposal bag, with an appropriate disinfectant, e.g. 0.5\% sodium hypochlorite.$^{36}$

4. Seal the bag; spray the outside with the disinfectant to wet all surfaces.

5. Remove gloves (and other PPE according to clinic policy).

6. Wash hands.

7. Ideally wait 24 hours before disposal.

\subsection{Disinfection of Miscellaneous Items}

The following methods of disinfection may be considered. (See section 4.1 Technique for using Disinfectant and section 4.2 Selection of a Disinfectant)

\section{i. Paper:}

Time: leave for 3 days in a well-ventilated place; dry heat: maintain at $100 \mathrm{C}$ for 15 minutes in an oven; moist heat - autoclave, ink may run; spray or immerse in liquid disinfectant - may destroy the paper, ink may run; UV/sunlight: expose paper to direct sunlight for 2-3 hours each side. ${ }^{14}$

ii. Money:

Time: store for 9 days in a clean place. Dry and moist heat may be used as for paper. Money generally tolerates these methods well. Disinfectants may be tried e.g. $70 \%$ alcohol spray/immersion, and immersion in dilute (up to $0.05 \%$ ) sodium hypochlorite solutions. ${ }^{14}$

iii. Pens:

Time: leave for 9 days. Disinfectants: Plastic pens may be washed with soap, and a disinfectant applied e.g. alcohol, hydrogen peroxide (not sodium hypochlorite).

iv. Cardboard boxes:

Time: Store in a clean well-ventilated area for 2-3 days before opening. Spraying with a disinfectant may be attempted in case urgent opening is required. Discard the box after opening and perform hand hygiene. ${ }^{14}$

v. Mobile phones:

Use a hand disinfectant or a disposable wipe soaked in disinfectant. ${ }^{14}$

vi. Keys:

Carry only essential keys. Disinfect using a hand disinfectant, or a disinfectant wipe/spray. May be periodically washed with soap and water.

\section{CONCLUSION}

Based on the needs of Pakistani dentists during the COVID-19 pandemic, guidelines established in this article 
include recommendations for clinic reorganization, improved ventilation, management of walk-in patients, correct use of PPE, environmental infection control, and protocols for aerosol procedures and disinfection. These recommendations supplement the Government of Pakistan guidelines for dental practice during the pandemic. Implementation of these guidelines requires effort at the national level for DHCW training, monitoring of practices, and ensuring availability of infection control supplies, as discussed in part 1 of this study.

\section{ACKNOWLEDGEMENTS}

The authors would like to acknowledge the help of Dr. Saqib Riaz Qazi and Dr. Maryam Ejaz in reviewing the article.

\section{CONFLICT OF INTEREST}

None declared

\section{REFERENCES}

1. Ministry of Health Services, Government of Pakistan. Guidelines for providing dental care services during COVID-19. 2020 May 3.

2. American Dental Association. Return to work interim guidance toolkit. 2020 Mar 24 [cited 2020 May 20]. Available from: https://www.ada.org/en/publications/ada-news/2020-archive/april/adatask-force-assembles-interim-guidance-toolkit-for-dentists-returningto-work

3. National Health Service. COVID-19 guidance and standard operating procedure. 2020 Apr 15 [cited 2020 May 20]. Available from: https://www.england.nhs.uk/coronavirus/wp-content/ uploads/sites/52/2020/04/C0282-covid-19-urgent-dental-care-sop.pdf

4. Alharbi A, Alharbi S, Alqaidi S. Guidelines for dental care provision during the COVID-19 pandemic. The Saudi Dent J. 2020 Apr 7. https://doi.org/10.1016/j.sdentj.2020.04.001

5. California Department of Public Health. COVID-19 guidance for dentistry. 2020 Apr 7 [cited 2020 May 20]. Available from: https://www.cdph.ca.gov/Programs/CID/DCDC/Pages/COVID-19/ GuidanceforDentistry.aspx

6. Missouri COVID-19 Dental Task Force. Guidance for your practice. 2020 Apr 29 [cited 2020 May 20]. Available from: https://sitefinity.ada.org/docs/librariesprovider30/publications/covid/ task-force-guidelines-for-mo-dental-practices_042920.pdf?sfvrsn=2

7. Indian Dental Association. Indian Dental Association's preventive guidelines for dental professionals on the coronavirus threat. 2020 [cited 2020 May 20]. Available from: https://www.ida.org.in/ pdf/IDA_Recommendations_for_Dental_Professionals_on_the_Cor onavirus_Threat.pdf
8. Center for Disease Control and Prevention. Interim infection prevention and control guidance for dental settings during the COVID19 response. 2020 May 3 [cited 2020 May 20]. Available from: https://www.cdc.gov/coronavirus/2019-ncov/hcp/dental-settings.html

9. Lynne Sehulster, Raymond Y.W. Chinn. Guidelines for environmental infection control in health-care facilities, recommendations of CDC and the Healthcare Infection Control Practices Advisory Committee (HICPAC). 2003 / 52(RR10);1-42

10. World Health Organization. COVID-19: Operational guidance for maintaining essential health services during an outbreak. 2020 March 25 [cited 2020 May 20]. Available from: https://www.who.int/ emergencies/diseases/novel-coronavirus-2019/technicalguidance/infection-prevention-and-control

11 William G. Kohn, Amy S. Collins, Jennifer L, Jennifer A. Harte, Kathy J, Dolores M. Malvitz. Guidelines for infection control in dental health-care settings. Morbidity and Mortality Weekly Report Recommendations and Reports Vol 52. 2003; No. RR-17

12.Centers for Disease Control and Prevention. Guidelines for environmental infection control in health-Care facilities. 2003 [cited 2020 May 20]. Available from: https://www.cdc.gov/infectioncontrol/ guidelines/environmental/appendix/air.html

13. Qazi SR, Qureshi A, Nasir S, Khan A. Cross infection control in dental practice - guiding manual for dental health care workers, World Health Organization (WHO)/ Ministry of Health, Government of Pakistan, Publication, Islamabad. 2005.

14. van Doremalen N, Bushmaker T, Morris DH, Holbrook MG, Gamble A, Williamson BN et al. Aerosol and surface stability of SARS-CoV-2 as compared with SARS-CoV-1. New England Journal of Medicine. 2020;382:1564-67.

https://doi.org/10.1056/NEJMc2004973

15. Gore B, Smith K. Tuberculosis infection control: a practical manual for preventing TB, 2011. San Francisco (CA). 2011.

16. Anderson J, Geeslin A, Streifel A. Minnesota Department of Health Office of Emergency Preparedness. Airborne infectious disease management: methods for temporary negative pressure isolation. St. Paul, MN.

17. Liu Y, Ning Z, Chen Y, Guo M, Liu Y, Gali NK et al. Aerodynamic analysis of SARS-CoV-2 in two Wuhan hospitals. Nature. $2020 \mathrm{Apr}$ 27:1-6

https://doi.org/10.1038/s41586-020-2271-3

18. Andersen BM, Bånrud H, Bøe E, Bjordal O, Drangsholt F. Comparison of UV C light and chemicals for disinfection of surfaces in hospital isolation units. Infection Control \& Hospital Epidemiology. 2006;27:729-34.

https://doi.org/10.1086/503643

19. American Dental Hygienists' Association. ADHA interim guidance on returning to work. 2020 May 28 [cited 2020 May 28]. Available from: https://www.adha.org/resources-docs/ADHA_Task ForceReport.pdf 
20. World Health Organisation. How to put on and take off personal protective equipment (PPE). 2020 April 22 [cited 2020 May 20]. Available from: https://www.who.int/csr/resources/publications/ putontakeoffPPE/en/

21. U.S. Department of Health and Human Services, Center for Disease Control and Prevention, National Institute for Occupational Safety and Health, DHHS (NIOSH). How to properly put on and take off a disposable respirator. 2010-133

22. Cochran MA, Miller CH, Sheldrake MA. The efficacy of the rubber dam as a barrier to the spread of microorganisms during dental treatment. J Am Dental Association. 1989;119:141-44. https://doi.org/10.14219/jada.archive.1989.0131

23. Bentley CD, Burkhart NW, Crawford JJ. Evaluating spatter and aerosol contamination during dental procedures. J Am Dental Association (1939). 1994;125:579-84.

https://doi.org/10.14219/jada.archive.1994.0093

24. Peng X, Xu X, Li Y, Cheng L, Zhou X, Ren B. Transmission routes of 2019-nCoV and controls in dental practice. International Journal of Oral Science. 2020;12:1-6.

https://doi.org/10.1038/s41368-020-0075-9

25. Avasth A. High volume evacuator HVE in reducing aerosol an exploration worth by clinicians. Journal of Dental Health, Oral Disorders \& Therapy. 2018;9(9). https://doi.org/10.15406/jdhodt.2018.09.00371

26. Atkins J, Chartier Y, Pessoa-Silva CL, Jensen P, Li Y, Seto WH. Natural ventilation for infection control in health-care settings. Geneva: World Health Organization; 2009. Available from: https://www.who.int/water_sanitation_health/publications/natural_v entilation/en/

27. Leas BF, Sullivan N, Han JH, Pegues DA, Kaczmarek JL, Umscheid CA. Environmental cleaning for the prevention of healthcare-associated infections.

28. United States Environmental Protection Agency. List N: products with emerging viral pathogens and human Coronavirus claims for use against SARS-CoV-2. 2020 [cited 2020 May 20] Available from: https://www.epa.gov/pesticide-registration/list-n-disinfectants-useagainst-sars-cov-2
29. Kampf G, Todt D, Pfaender S, Steinmann E. Persistence of coronaviruses on inanimate surfaces and its inactivation with biocidal agents. J Hospital Infection. 2020 Feb 6.

https://doi.org/10.1016/j.jhin.2020.01.022

30. Ipswich Massachusetts Government. Guidelines on the use of disinfectants. 2011 Oct 31 [cited 2020 May 20]. Available from: https://www.ipswichma.gov/DocumentCenter/View/10072/50ppmGuidelines-on-the-Use-of-Disinfectants

31. Ribeiro MM, Neumann VA, Padoveze MC, Graziano KU. Efficacy and effectiveness of alcohol in the disinfection of semi-critical materials: a systematic review. Revista latino-americana de enfermagem. 2015;23:741-52.

https://doi.org/10.1590/0104-1169.0266.2611

32. Singh V, Nagaraja C, Hungund SA. A study of different modes of disinfection and their effect on bacterial load in dental unit waterlines. European J General Dentistry. 2013;2:246-51.

https://doi.org/10.4103/2278-9626.115999

33. Dallolio L, Scuderi A, Rini MS, Valente S, Farruggia P, Sabattini $\mathrm{M}$ et al. Effect of different disinfection protocols on microbial and biofilm contamination of dental unit waterlines in community dental practices. International journal of environmental research and public health. 2014;11:2064-76.

https://doi.org/10.3390/ijerph110202064

34. Fritz C. How to shock your dental waterlines. ProEdge Dental Water Labs, 2019 Mar 14 [cited 2020 May 20]. Available from: https://blog.proedgedental.com/blog/how-to-shock-your-dentalwaterlines

35. Chin A, Chu J, Perera M, Hui K, Yen HL, Chan M, Peiris M, Poon L. Stability of SARS-CoV-2 in different environmental conditions. medRxiv. 2020 Jan 1.

https://doi.org/10.1101/2020.03.15.20036673

36. Environmental Health and Safety, University of Toronto. Laboratory hazardous waste management and disposal manual. [Cited 2020 May 20]. Available from: https://ehs.utoronto.ca/laboratory-hazardouswaste-management-and-disposal-manual/biological-wastedisposal/using-bleach-as-a-disinfectant/ 